\title{
Effects of segmented electrode in Hall current plasma thrusters
}

\author{
Y. Raitses ${ }^{\text {a) }}$ \\ Princeton University Plasma Physics Laboratory, P. O. Box 451, Princeton, New Jersey 08543 \\ M. Keidar \\ Aerospace Engineering, University of Michigan, Ann Arbor, Michigan 48109 \\ D. Staack and N. J. Fisch \\ Princeton University Plasma Physics Laboratory, P. O. Box 451, Princeton, New Jersey 08543
}

(Received 26 November 2001; accepted 6 August 2002)

\begin{abstract}
Segmented electrodes placed along a ceramic channel in a Hall thruster are shown to influence significantly the plasma potential distribution. Both the radial potential and the axial acceleration region are sensitive to the location of the segmented electrodes. The measured and theoretical potential profiles appear to be affected in detail by the electrode material (graphite) having lower secondary electron emission than the ceramic channel walls. The measured plasma potential profile is shown as well to correlate with the observed and desirable narrowing of the plasma plume emanating from the thruster. (c) 2002 American Institute of Physics. [DOI: 10.1063/1.1510556]
\end{abstract}

\section{INTRODUCTION}

A conventional Hall current plasma thruster is a crossed field discharge device with a coaxial channel, in which the electrostatic acceleration of ions takes place in quasineutral plasma. A significant axial electric field is established in the vicinity of the maximum radial magnetic field, typically near the thruster exit. Thruster performance, namely, jet velocity, acceleration efficiency. and beam divergence, are strongly affected by the location of the acceleration region and therefore by the location of main axial electric field. The length of the acceleration region and its location relative to the magnetic field distribution depends mainly on the electron mobility and can be strongly affected by material properties of the channel walls and plasma-wall interaction. ${ }^{1-5}$ Generally speaking, a simple exchange of the channel materials, for example, from ceramic to metal, may lead to a different acceleration regime or at least to a different spatial distribution of plasma properties in the channel, ${ }^{2,3,5}$ even if not necessarily to changes in thruster performance. A possible reason for the thruster performance being similar is that at a given discharge voltage a change in material properties of the entire channel walls, namely the secondary electron emission (SEE) and conductivity, causes a change in the ion flux to the walls, which is balanced by a change in a length of the acceleration region. As a result, there are no substantial differences of the ion wall losses and thus, the integral characteristics of the thruster. ${ }^{2,3}$

The conventional Hall thruster channel is made entirely from either ceramic or metal. However, in a segmented electrode Hall thruster, metal electrodes are placed along the ceramic channel. It was shown in Refs. 6 and 7 that a Hall thruster of single- and multisegmented electrode configurations was able to reduce the exhaust plasma plume angle by about $20 \%$ compared to a conventional nonsegmented

a) Author to whom correspondence should be addressed; electronic mail: yraitses@pppl.gov thruster configuration. The mechanism that leads to the plume narrowing, as well as to the generally large plume angle $\left(\sim 90^{\circ}\right)$ for Hall thrusters in the first place is not clear yet. The object of the present study is to examine in detail how the plasma responds to the presence of a segmented electrode in the ceramic channel.

This article is organized as following: The experimental setup and results are described in Sec. I. Section II devoted to the description of the theoretical approach and important results of the simulation. In Secs. III and IV we compare and discuss the measured and predicted results.

\section{EXPERIMENTAL SETUP AND RESULTS}

The $9 \mathrm{~cm}$ laboratory Hall thruster and test facility used in this study has been described in detail in Refs. 6 and 7. Figure 1 shows this thruster, with a $4 \mathrm{~mm}$ length segmented electrode placed, on the inner wall near the exit of the channel. The channel is made of boron nitride ceramic and the segmented electrode is low sputtering carbon-carbon-fiber graphite material. The placement of the electrode relative to the magnetic field distribution is shown in Fig. 2. As shown in Ref. 6, this placement of a single segmented electrode corresponds to the most substantial reduction of the plume angle. Moreover, in a different set of experiments with a two-segmented electrode configuration, an additional graphite electrode of $8 \mathrm{~mm}$ length was placed on the outer channel wall opposite to the inner segmented electrode.

In these experiments, we used a fast movable emissive probe setup to measure the plasma potential distribution inside the thruster channel (Fig. 1). The floating potential of the probe relative to ground was measured using a 1:100 isolating amplifier and monitored by a personal computer (PC)-based data acquisition system. The details of the emissive probe setup are described in Ref. 8. In the present experiments, the residence time of the probe inside the channel including probe insertion and removal was shorter than $0.3 \mathrm{~s}$. The probe positions relative to the thruster channel was mea- 


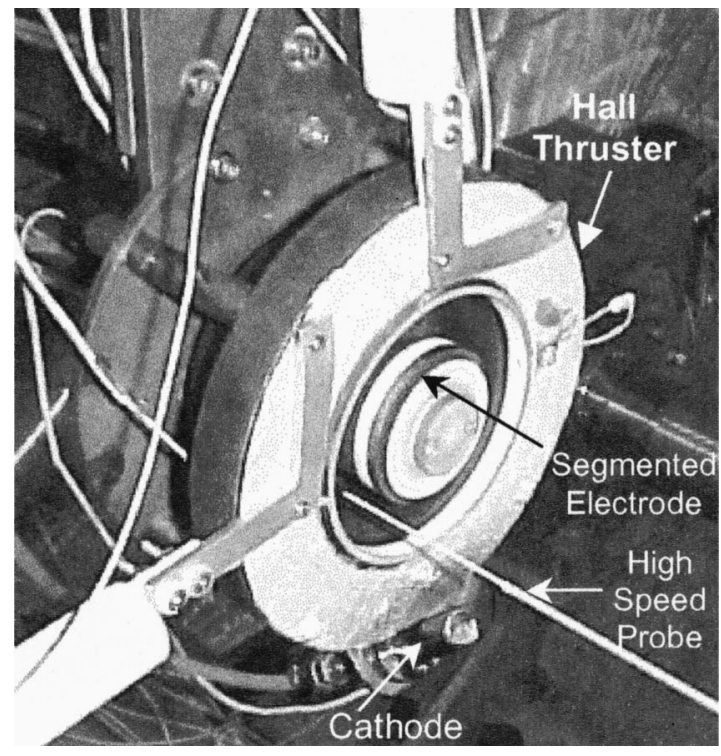

FIG. 1. Segmented electrode Hall thruster and probe setup.

sured by a Rensishaw optical encoder with $20 \mu \mathrm{m}$ resolution. Control and measurement of this positioning system is performed by a PC-based data acquisition system. Other measurements included mass flow rate, discharge current, voltage, and electromagnetic coils current. The segmented electrodes were floating relative to ground.

The thruster was operated in nonsegmented, single segmented and two-segmented configurations. Potential profiles were measured for nine radial positions of the probe. At each radial position, the heater power to the emissive probe was gradually increased until the floating potential reached saturation values along the probe traveling path. Reproducibility of probe measurements was greater than $85 \%$. The major source of irreproducibility was probe-induced perturbations of the thruster discharge current, which tended to increase and then saturate as the probe moved toward the anode (Fig. $3)$. The amplitude of the discharge current perturbations could reach $50 \%$ of its steady state value. These perturbations appear less than $0.1 \mathrm{~s}$ after the probe immersion and are more substantial at the channel median than near the outer and inner walls. Therefore, it is not clear if they result from

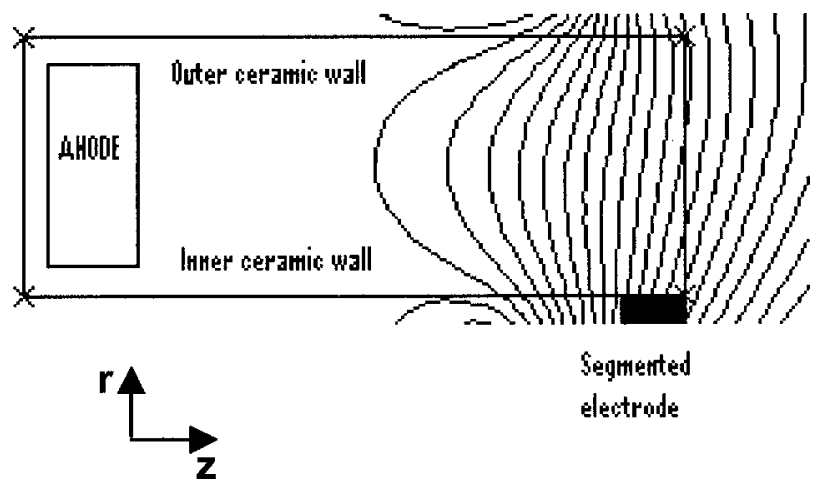

FIG. 2. Magnetic field distribution, coordinate system and placement of the graphite segmented electrode on the inner wall of the coaxial thruster channel and the anode (a half view relative to the thruster axis $z$ ).

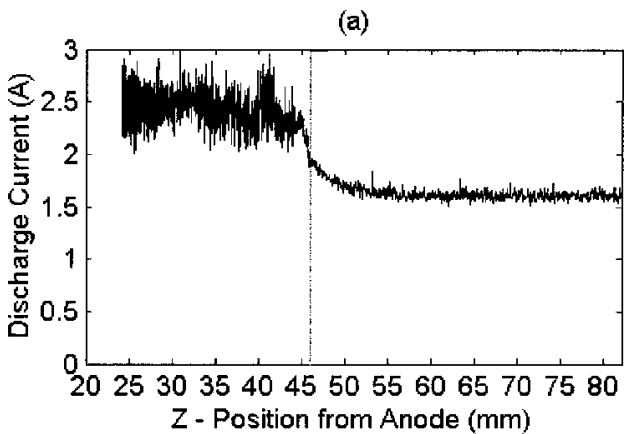

(b)

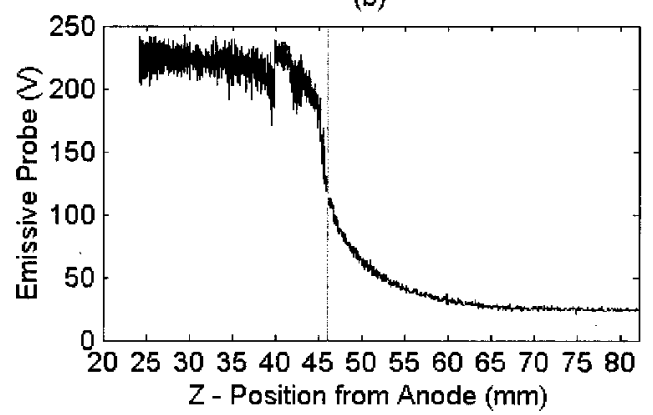

FIG. 3. Discharge current (a) and probe floating potential (b) vs the probe position relative to the anode. The traces were measured during probe insertion in the thruster plasma along the channel median. The channel exit is at $46 \mathrm{~mm}$.

probe heating and ablation by electron Hall current. ${ }^{9}$ In addition to these perturbations, uncertainties of the emissive probe measurements include a voltage drop across the filament produced by the dc heating power supply $(\sim 10 \mathrm{~V})$ and a double sheath formed between the hot floating probe and plasma $\left(\Delta \varphi \approx T_{e}\right){ }^{10}$

Figure 4 shows equipotential counter lines derived from measurements for single segmented and nonsegmented thruster configurations. These results were measured at the same operating conditions: discharge voltage of $250 \mathrm{~V}$ and Xenon gas mass flow rate of $1.7 \mathrm{mg} / \mathrm{s}$. The magnetic field was also constant. Remarkably, the location of the acceleration and ionization region relative to the magnetic field distribution is substantially affected by the single segmented electrode. It moves a few millimeters upstream of the channel, as compared to nonsegmented thruster configuration. As a result, there is a relatively smaller fraction of the voltage drop left outside the thruster channel in the fringing magnetic field. In the conventional configuration, about $40 \%-50 \%$ of the measured voltage drop is outside the thruster channel. As can be seen from the model described below, this effect of the segmented electrode can be partially attributed to the dependence of the plasma flow on the SEE of the wall materials.

\section{MODEL OF THE PLASMA FLOW IN THE HALL THRUSTER WITH SEGMENTED ELECTRODE}

In this section we describe the model of the plasma flow in an annular channel as shown in Fig. 2. The plasma flow starts in the near anode region and the lateral boundaries of the plasma flow region is the entrance to the sheath at the dielectric walls. The entire quasineutral plasma region is 
(a) Non-Segmented

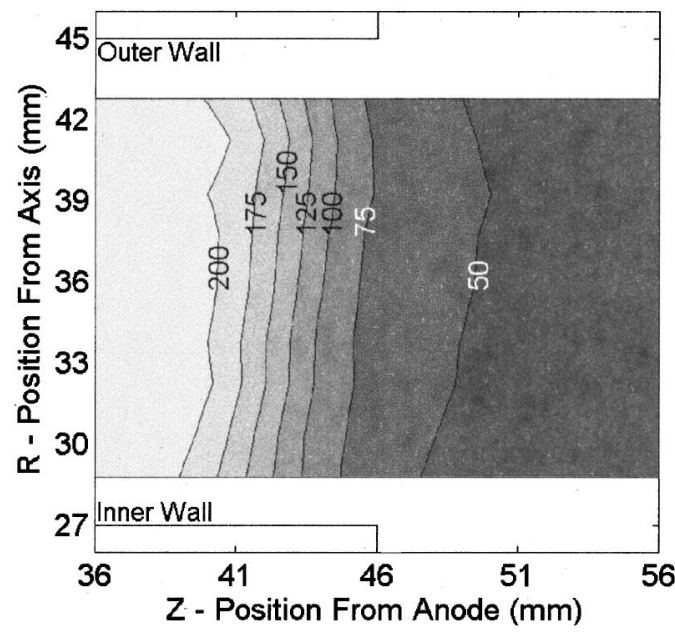

(b) Inner Segmented Electrode

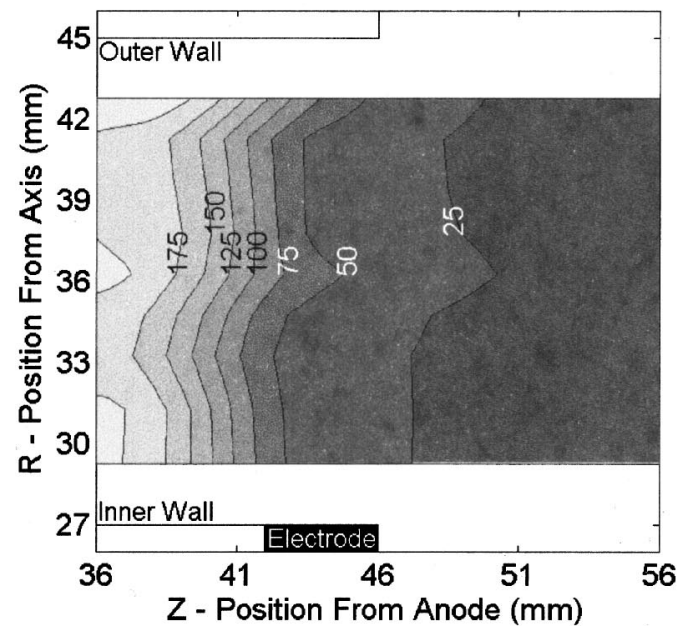

FIG. 4. Plasma potential distribution measured relative to ground for nonsegmented (a) and segmented (b) thruster configurations at the same operating conditions. The electrode made from a graphite material is placed on the inner channel wall. The channel exit is at $46 \mathrm{~mm}$. The inner wall at $R_{\text {in }}=27 \mathrm{~mm}$ and outer wall at $R_{\text {out }}=45 \mathrm{~mm}$.

considered as a presheath in which the boundary conditions for the sheath entrance are developed. Details of the model of the plasma-sheath transition region are described elsewhere. ${ }^{3}$ The sheath region in front of the dielectric surface is considered to be collisionless. The dielectric wall effect is taken into account by introducing an effective coefficient of SEE, $s$. Segmented metal electrode is introduced in the model as a region with smaller SEE as shown in Fig. 2.

A plasma is considered with "magnetized" electrons and "unmagnetized" ions, i.e., $\rho_{e} \ll H \ll \rho_{i}$, where $\rho_{e}$ and $\rho_{i}$ are the Larmor radii for the electrons and ions, respectively, and $H$ is the channel characteristic length. It is assumed that the magnetic field has only a radial component. Cylindrical coordinates will be used, as shown in Fig. 2, with angle $\theta$, radius $r$, and axial distance from the anode $z$, respectively. A hydrodynamic model is employed in a two-dimensional (2D) domain assuming that the system reaches a steady state. The momentum and mass conservation equations for electrons, ions and neutrals under these conditions have the following form:

$$
\begin{aligned}
& n m_{i}\left(\mathbf{V}_{\mathbf{i}} \boldsymbol{\nabla}\right) \mathbf{V}_{\mathbf{i}}=n e \mathbf{E}-\nabla P_{i}-\beta n m_{i} n_{a}\left(\mathbf{V}_{\mathbf{i}}-\mathbf{V}_{\mathbf{a}}\right), \\
& 0=-e n(\mathbf{E}+\mathbf{V} \times \mathbf{B})-\nabla P_{e}-n \nu_{\mathrm{ef}} m_{e}\left(\mathbf{V}_{\mathbf{e}}-\mathbf{V}_{\mathbf{i}}\right), \\
& \nabla \cdot\left(V_{i} n\right)=\beta n n_{a}, \\
& \nabla \cdot\left(V_{a} n_{a}\right)=-\beta n n_{a},
\end{aligned}
$$

where $i, a$, and $e$ are subs for ions, neutral atoms and electrons, respectively, $n$ is the plasma density, $\beta$ is the ionization rate, $V$ is the velocity, $\nu_{\text {ef }}$ is the effective collision frequency.

In order to simplify the problem, yet including the major physical effects, we consider one-dimensional flow of the neutrals. Since only the radial magnetic field component is considered in the model, the electron transport is greater in the azimuthal direction $(E \times B$ drift $)$ than in the axial direction (drift diffusion due to collisions). Electrons can freely move along magnetic field lines and therefore the electric field potential is constant along magnetic field lines within accuracy of the electron pressure gradient. If we assume that the electron temperature is constant along each magnetic field line we obtain

$$
\varphi-\frac{k T_{e}}{e} \ln (n)=\text { const. }
$$

The left-hand side of this equation is known as a thermalized potential. ${ }^{1}$ This equation makes it possible to reduce the twodimensional calculation of the electric field to a onedimensional problem in a way similar to Refs. 3, 11, and 12 . Calculating the potential distribution along the channel centerline makes it possible to calculate the potential in the entire domain. The electron temperature is calculated along the centerline as a balance between the Joule heating, ionization and wall losses as described elsewhere. ${ }^{3,11}$ In this model we consider that electron transport across the magnetic field is due several collision mechanisms: electron-neutral collisions, electron-wall collisions, and anomalous (Bohm) diffusion: $\nu_{\mathrm{ef}}=\nu_{\mathrm{en}}+\nu_{\mathrm{ew}}+\nu_{B}$, where $\nu_{\mathrm{ef}}$ is the effective electron collision frequency.

At the upstream boundary (anode plane, $z=0$, see Fig. 2) we specify the density and velocity similarly to Ref. 1 assuming that the ion velocity $V_{0}=2 \times 10^{3} \mathrm{~m} / \mathrm{s}$ near the anode that corresponds to $3 \mathrm{eV}$. This upstream condition implies that we are considering only supersonic plasma flow assuming that the transition from the subsonic to supersonic flow occurs in the anode vicinity. Recent analyses of the nearanode region confirm this assumption. ${ }^{13}$ The atom velocity near the anode is assumed to be $V_{\mathrm{oa}}=2 \times 10^{2} \mathrm{~m} / \mathrm{s} .{ }^{14}$ At the downstream boundary (thruster exit plane, $z=L$ ), we specify electron temperature, assuming, similarly to Ref. 14, that $T_{e}=5 \mathrm{eV}$ which is close to that measured in the experiment. ${ }^{15}$ Results are presented for the following conditions: Discharge current: 1.65 A (segmented electrode thruster case) and $1.6 \mathrm{~A}$ (conventional thruster case), anode mass flow rate $1.7 \mathrm{mg} / \mathrm{s}$. We used the magnetic field axial profile corresponding to the measured value along the centerline of the channel. 


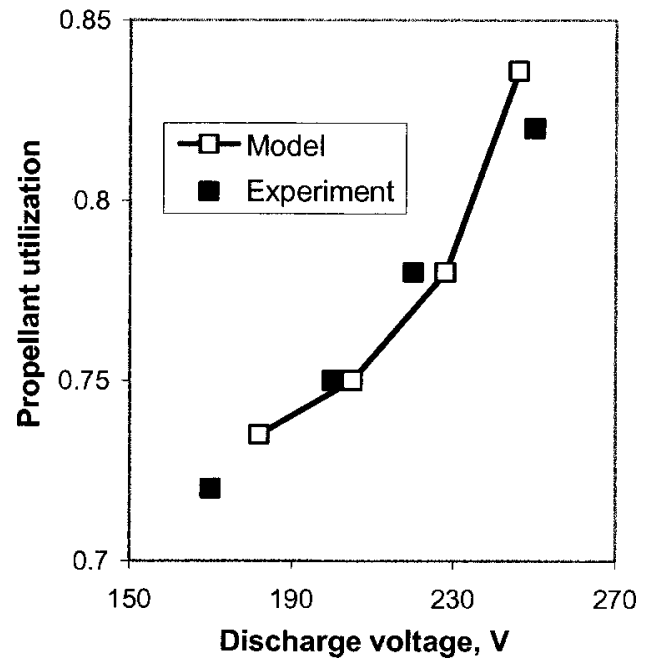

FIG. 5. Calculated propellant utilization at the thruster exit plane and comparison with measurements of Ref. 6 .

The numerical analysis is similar to that developed previously. ${ }^{16}$ We use the implicit two-layer method to solve the system of Eqs. (1)-(4). These equations are approximated by a two-layer six point scheme. The electron temperature distribution in the axial direction is calculated by iteration. More details about this model and numerical solution can be found in Ref. 3 .

Calculated propellant utilization at the thruster exit plane $x=L$ is shown in Fig. 5. For comparison the measured propellant utilization is also shown. ${ }^{6}$ One can see that the magnitude as well as general trend predicted by the model are close to that observed experimentally. It should be noted that the same agreement was obtained for the thrust.

Axial distribution of the electron temperature is shown in Fig. 6. It can be seen that the electron temperature is higher in the case of the channel with segmented electrode and peaks at about $18 \mathrm{eV}$ in the acceleration region near the channel exit (the peak corresponds to the segmented electrode position).

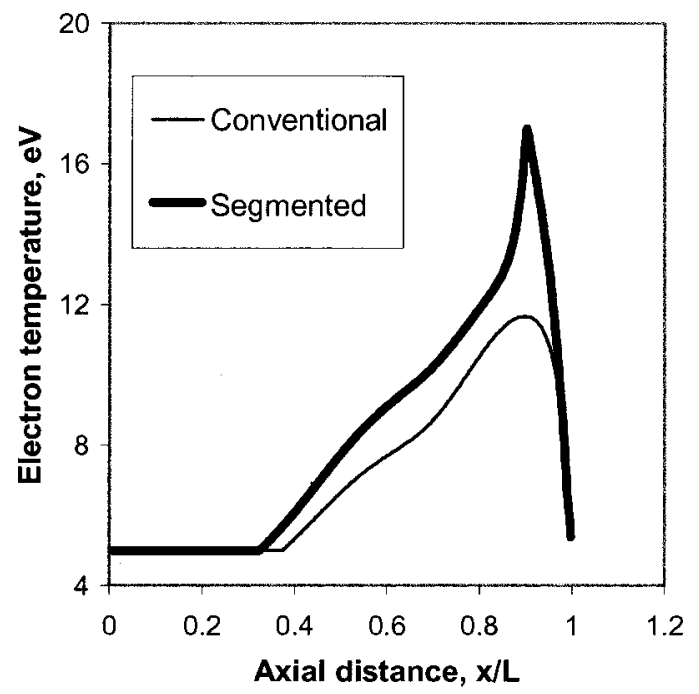

FIG. 6. Calculated axial distribution of the electron temperature. $\mathrm{L}$ is the channel length taken from the anode to the thruster exit.

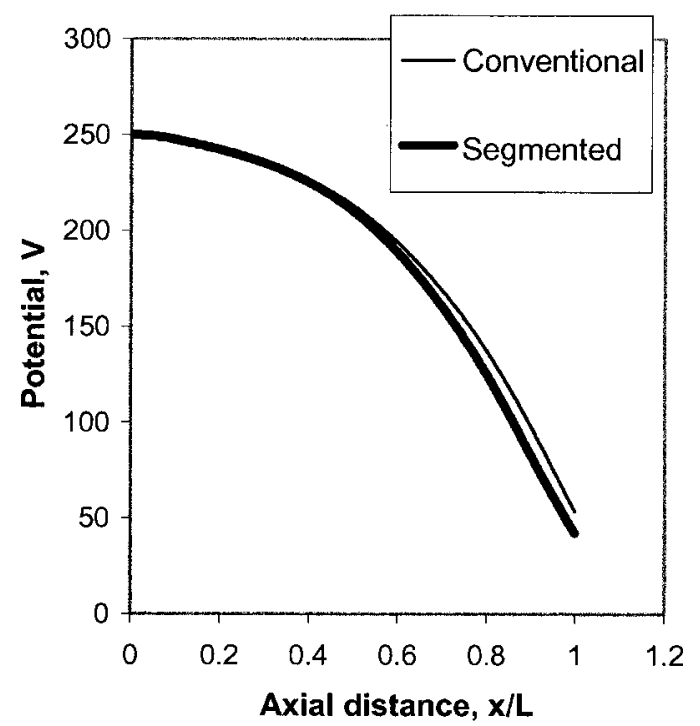

FIG. 7. Calculated potential distribution along the channel median for two thruster configurations. $L$ is the channel length taken from the anode to the thruster exit.

The potential distribution along the channel centerline for two thruster configurations is shown in Fig. 7. In the case of segmented electrode the potential is low by about $15 \mathrm{~V}$ at the exit plane. In these calculations we employed the experimental fact ${ }^{6,7}$ that the discharge current is larger in the case of the thruster operated with segmented electrode.

\section{DISCUSSION}

We shall now discuss and compare our model prediction of plasma potential distribution with experimental results. We will focus on two major experimentally observed effects: the inward shift of the plasma potential distribution and the unexpected plasma potential structure in the radial direction for segmented electrode thruster case as compared to conventional case (Fig. 4).

Both the experimental results and the numerical simulation show a shift in the acceleration region towards the anode (Figs. 4 and 7). In other words the potential at the exit plane is lower in the case of the segmented electrode. In the model, we took into account the experimental fact that the discharge current is larger in the segmented configuration. A larger discharge current leads to the solution with higher electric field and therefore higher potential drop across the channel. However the magnitude of the shift in the model is less than the measured shift. Possible reasons for the discrepancy could be both experimental and theoretical. For example, the probeinduced perturbations of the plasma potential could be different in the segmented electrode configuration than in the conventional thruster, though the perturbations to the discharge current were similar in both thruster configurations. On the other hand the theoretical model does not take into account the 2D configuration of the magnetic field nor does it take into account the ion collecting role of the electrode. Additionally the model employs local balance of the ion and electron fluxes to the wall of segmented electrode while in 


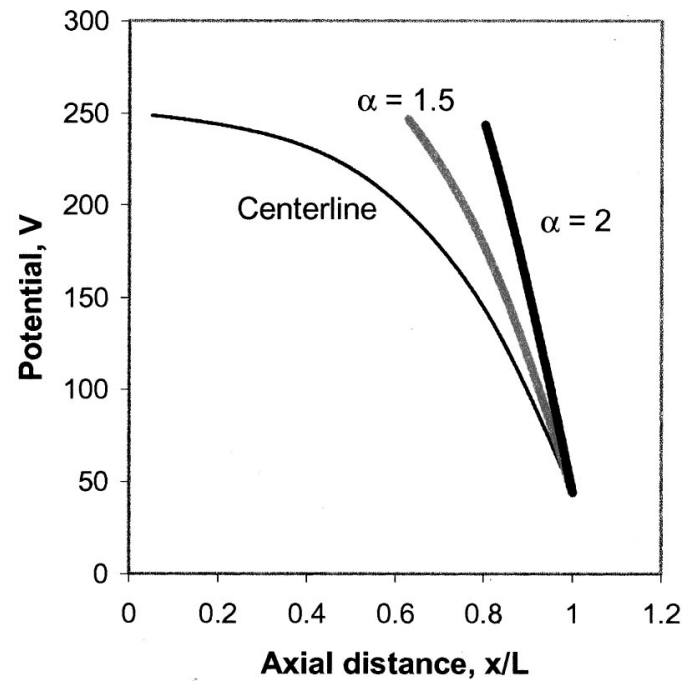

FIG. 8. Calculated plasma potential profiles for different ratios of the axial electron current. $L$ is the channel length taken from the anode to the thruster exit.

reality the only global balance of the ion and electron flux (i.e., integrated over the length of the electrode) has taken place.

In the present model we assumed that the electron temperature is constant in the radial direction, i.e., along magnetic field lines, and therefore the axial distribution of the electron temperature shown in Fig. 6 has the meaning of some average $T_{e}$ in the channel cross section. However, in reality, the electron temperature field in the Hall thruster channel is rather two dimensional. Following results of Fig. 6 and taking into account that metals have typically smaller SEE than ceramics at the same energy of primary electrons, one can expect that the electron temperature will be higher near the graphite segment placed in the boron nitride channel. On the other hand, there is no segmented electrode on the opposite side of the channel (See Fig. 2). As a result, the presence of the segmented electrode only on one side of the channel may create an electron temperature gradient in the radial direction and lead to electron current along the magnetic field lines $j_{e} \|$. Then, current conservation implies that the electron current in the axial direction near the ceramic wall opposite to the segmented electrode must increase. Using the plasma parameter distribution from our 2D fluid simulation, a possible potential distribution near the outer wall can be calculated assuming that the current density there is higher than that in the middle of the channel. In this case, the potential distribution depends on the parameter $\alpha$, which is defined as ratio of the axial electron current density near the wall to the axial electron current density along the midway. Illustrative results of these calculations for different $\alpha$ are shown in Fig. 8. Here we assumed that the potential distribution in the radial direction at the thruster exit plane $(z=L)$ is uniform. As can be seen, higher current density leads to steeper potential distribution.

Note, that a nonuniform distribution of the axial electron current density in the radial direction may be responsible for unusual concave shape of equipotentials measured for the segmented electrode case (Fig. 4). Indeed, Fig. 9 compares

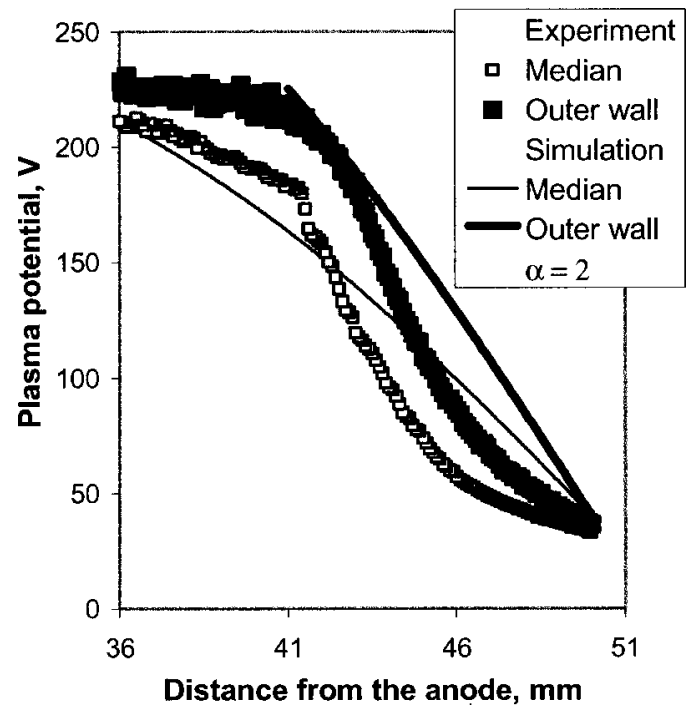

FIG. 9. Measured and calculated potential profiles along the channel median and outer wall for the segmented electrode case. The channel exit is at 46 $\mathrm{mm}$.

measured and calculated potential profiles along the channel median and near the outer wall. Assuming electron current density near the outer wall is higher than the axial electron current density at the middle we can get a qualitative agreement between theory and experiment. The reasonable agreement is obtained in the case of $\alpha=2$ as shown in Fig. 9. In addition, these theoretical predictions are also supported by the results measured for two segmented electrode configuration with an additional electrode placed on the outer wall of the ceramic channel, opposite to the inner segmented electrode (Fig. 10). In this thruster case, no concave shape of equipotential was observed and the measured plasma potential changes are insignificant in the radial direction as compared to the single segmented electrode configuration of Fig.

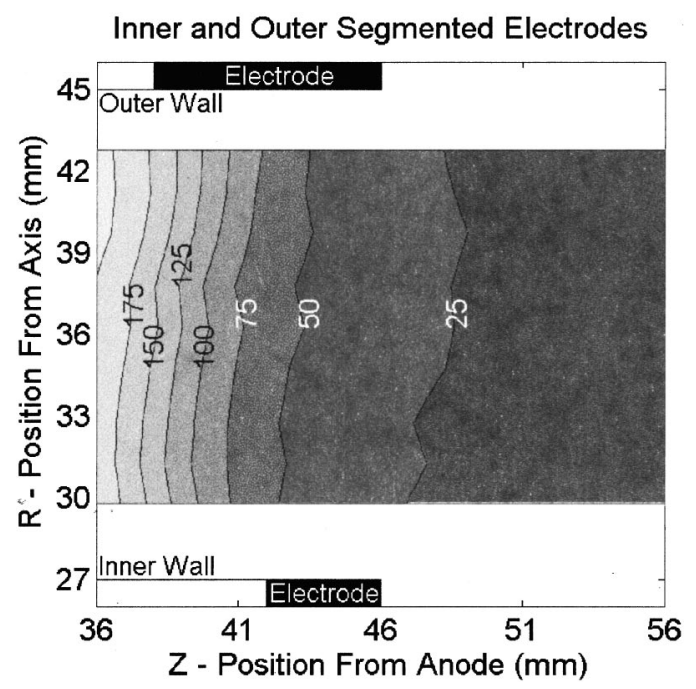

FIG. 10. Plasma potential distribution measured for two-segmented thruster configuration. An additional $8 \mathrm{~mm}$ length segmented electrode is placed on the outer wall near the exit opposite to the inner segmented electrode. Both electrodes are made from a graphite material. The channel exit is at $46 \mathrm{~mm}$. The inner wall at $R_{\text {in }}=27 \mathrm{~mm}$ and outer wall at $R_{\text {out }}=45 \mathrm{~mm}$. 
4. These results suggest that electron temperature gradient in the radial direction may be responsible for developing the unusual concave shape of equipotentials observed in these experiments with single segmented electrode.

\section{CONCLUDING REMARKS}

A segmented electrode Hall thruster channel leads to some interesting phenomena measured experimentally and partially explained by a hydrodynamic plasma model. Two major effects of the segmented electrode include the inward shift of the acceleration region and the changes in the shape of equipotentials. These effects might be due to a lower secondary electron emission of the segmented electrode as compared to ceramic channel walls. Therefore, the shape of equipotentials should depend strongly on physical properties of the channel materials, in particular, at intersections of the magnetic field lines with the outer and inner channel walls. On the other hand, conductivity of the segmented electrode, which was not considered in the present model, might also contribute to these effects by allowing a current along the floating electrode, while maintaining the global balance of electron and ion fluxes from the plasma. This current can result from locally unequal electron and ion fluxes collected by the equipotential surface of the electrode from the plasma with a voltage potential drop along the electrode. ${ }^{17}$

It is interesting to note that these results were obtained using only passive segmented electrodes (without electron emission from the electrodes). Such passive electrodes were proved to be an important tool for control plasma potential distribution in the Hall thruster channel. In addition, the model predicts and our recent experimental results have indicated, that use of segmented rings made from ceramic materials with different SEE properties instead of metal electrodes can also affect the plasma potential distribution. ${ }^{17} \mathrm{On}$ the other hand, the differences of SEE between metals and boron nitride ceramic are more substantial than between ceramics suitable for harsh thruster operating conditions. Therefore, it seems that segmented electrodes are still the preferable tool for control of plasma potential distribution.

\section{ACKNOWLEDGMENTS}

The authors wish to thank L. Dorf and A. Litvak for fruitful discussions and help with experiments. This work was supported by grants from the U.S. Department of Energy under Contract No. DE-AC02-76-CHO3073 and the New Jersey Science and Technology Commission.

${ }^{1}$ A. I. Morozov and V. V. Savelyev, in Review of Plasma Physics, edited by B. B. Kadomtsev and V. D. Shafranov (Consultants Bureau, New York, 2000), Vol. 21, p. 203

${ }^{2}$ V. V. Egorov, V. Kim, A. A. Semenov, and I. I. Shkarban, Ion Injectors and Plasma Accelerators (Energoizdat, Moscow, 1990), p. 56 (in Russian).

${ }^{3}$ M. Keidar, I. D. Boyd, and I. I. Beilis, Phys. Plasmas 8, 5315 (2001).

${ }^{4}$ A. Fruchtman, N. J. Fisch, and Y. Raitses, Phys. Plasmas 8, 1048 (2001).

${ }^{5}$ E. Y. Choueiri, Phys. Plasmas 8, 5025 (2001).

${ }^{6}$ Y. Raitses, L. A. Dorf, A. A. Litvak, and N. J. Fisch, J. Appl. Phys. 88, 1263 (2000).

${ }^{7}$ N. Fisch, Y. Raitses, L. A. Dorf, and A. A. Litvak, J. Appl. Phys. 89, 2040 (2001).

${ }^{8}$ Y. Raitses and N. J. Fisch, Phys. Plasmas 8, 2579 (2001).

${ }^{9}$ J. M. Haas and A. D. Gallimore, Rev. Sci. Instrum. 71, 4131 (2000).

${ }^{10}$ V. A. Rozhansky and L. D. Tsendin, Transport Phenomena in Partially Ionized Gases (Gordon and Breach, Amsterdam, 2000), Chap. 3.

${ }^{11}$ M. J. Fife and M. Martinez-Sanchez, 24th International Conference on Electric Propulsion, Moscow, Russia, 1995, IEPC Paper 95-240.

${ }^{12}$ I. D. Boyd, L. Garrigues, J. Koo, and M. Keidar, 36th AIAA Joint Prop. Conference, Huntsville, AL, 2000, Paper AIAA-2000-3520.

${ }^{13}$ M. Keidar, I. D. Boyd, and I. I. Beilis, The 38th AIAA Joint Prop. Conference, Indianapolis IN, July 2002, AIAA Paper -2002-4107.

${ }^{14}$ J. P. Bouef and L. Garrigues, J. Appl. Phys. 84, 3541 (1998).

${ }^{15}$ S. Kim, J. E. Foster, and A. D. Gallimore, AIAA Paper-96-2972, July 1996.

${ }^{16}$ M. Keidar, I. Beilis, R. L. Boxman, and S. Goldsmith, J. Phys. D 29, 1973 (1996).

${ }^{17}$ Y. Raitses, D. Staack, and N. J. Fisch, The 38th AIAA Joint Prop. Conference, Indianapolis, IN, July 2002, AIAA Paper-2002-3954. 